\title{
A Monolithic, FEM-Based Approach for the Coupled Squeeze Film Problem of an Oscillating Elastic Micro-Plate Using 3D 27-Node Elements
}

\author{
Anish Roychowdhury ${ }^{1,2^{*}}$, Arup Nandy ${ }^{1}$, C. S. Jog ${ }^{1}$, Rudra Pratap ${ }^{1,2}$ \\ ${ }^{1}$ Department of Mechanical Engineering, Indian Institute of Science, Bangalore, India \\ ${ }^{2}$ Center for Nano Science and Engineering, Indian Institute of Science, Bangalore, India \\ Email: *anishroy@mecheng.iisc.ernet.in
}

Received August 2013

\begin{abstract}
In this study we describe an FEM-based methodology to solve the coupled fluid-structure problem due to squeeze film effects present in vibratory MEMS devices, such as resonators, gyroscopes, and acoustic transducers. The aforementioned devices often consist of a plate-like structure that vibrates normal to a fixed substrate, and is generally not perfectly vacuum packed. This results in a thin film of air being sandwiched between the moving plate and the fixed substrate, which behaves like a squeeze film offering both stiffness and damping. Typically, such structures are actuated electro-statically, necessitating the thin air gap for improving the efficiency of actuation and the sensitivity of detection. To accurately model these devices the squeeze film effect must be incorporated. Extensive literature is present on modeling squeeze film effects for rigid motion for both perforated as well as non-perforated plates. Studies which model the plate elasticity often use approximate mode shapes as input to the 2D Reynolds Equation. Recent works which try to solve the coupled fluid elasticity problem, report iterative FEM-based solution strategies for the 2D Reynolds Equation coupled with the 3D elasticity Equation. In this work we present a FEM-based single step solution for the coupled problem at hand, using only one type of element ( 27 node 3D brick). The structure is modeled with 27 node brick elements of which the lowest layer of nodes is also treated as the fluid domain (2D) and the integrals over fluid domain are evaluated for these nodes only. We also apply an electrostatic loading to our model by considering an equivalent electrostatic pressure load on the top surface of the structure. Thus we solve the coupled 2D-fluid-3D-structure problem in a single step, using only one element type. The FEM results show good agreement with both existing analytical solutions and published experimental data.
\end{abstract}

Keywords: Squeeze Film Damping; Coupled Problem; 27-Node Brick; Micro-Plate

\section{Introduction}

The wide scale application of electro-statically driven MEMS sensors, using parallel plate capacitors have led to increasing interest in the study of energy dissipation due to the thin film of air trapped in such devices. Typically such devices consist of a plate like structure, vibrating normally to a fixed substrate, with a thin air film trapped in-between (see Figure 1). If the lateral dimensions of the plate happen to be much larger than the height of the air gap, the trapped air behaves both like a spring and a viscous damper, a phenomenon known as squeeze film effect. Squeeze film damping is the dominant dissipation mechanism in Si based MEMS devices operating in the aforementioned conditions [1]. The

*Corresponding author. squeeze film offered damping and stiffness changes the dynamic characteristics of the vibratory MEMS device [2]. In order to correctly model such devices, it is necessary to accurately determine the stiffness and damping due to the squeeze film. Accurate modeling of such systems involves coupling of three domains, electrostatics, structural and fluid. Traditionally the squeeze film domain effect is modeled using the lubrication theory, via

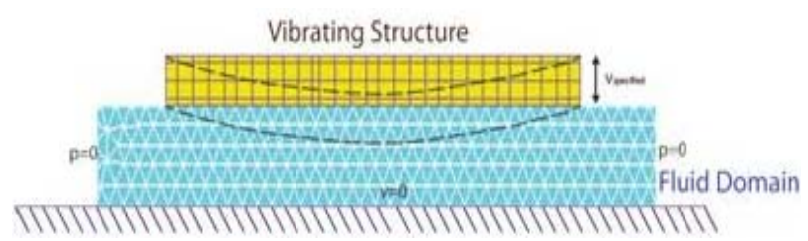

Figure 1. Schematic of an air film trapped between a vibrating elastic plate and a fixed substrate. 
the Reynolds Equation [3]. With rigid plate assumption the Reynolds Equation can be decoupled from the elasticity Equation and, further, on linearization can be solved to obtain analytical expressions for stiffness and damping. Blech [3] studied the effect of squeeze film induced stiffness and damping for rigid plates with trivial pressure boundary conditions. Darling et al. [4] presented analytical solutions to the linearized Reynolds Equation for various venting conditions, using a Greens function approach. For flexible structures one has to account for the variable air gap, and the elasticity Equation has to be coupled with the Reynolds Equation for accurate modeling. Hung et al. [5] presented a reduced order macro model based on basis functions generated from finite difference simulations. They applied this technique to model a pressure sensor as a clamped-clamped microbeam to study the pull-in dynamics of the system using a 1D Euler beam Equation and the non-linear Reynolds Equation. McCarthy et al. [6] studied cantilever microswitches using a transient finite difference method approximating a parabolic pressure distribution along the length and non variance along the width and obtained good agreement with experimental measurements. Younis et al. [7] studied the effect of squeeze film damping for an electrically actuated micro-plate, using the compressible Reynolds Equation. They used perturbation methods to derive analytical expressions for pressure distributions in terms of the structural mode shape. Pandey et $a l$. [8] studied the effect of flexural mode shapes on the squeeze film offered stiffness and damping for a cantilever resonator, they used Green's function to solve the linearized compressible Reynold's Equation and used the modal projection method available in ANSYS to solve the coupled fluid structure problem for several flexural modes of vibration. The analytical and numerical values of damping obtained were in good agreement with experimental results. Li et al. [8] accounted for the static bias deflection for a fixed- fixed micro-beam and a cantilever under electrostatic actuation. They assumed a parabolic function for the pressure along the beam width and a cosine series along the beam length, and solved the coupled Reynolds Equation and the Euler Bernoulli beam Equation. Hannot et al. [9] presented an approach to solve the coupled elasticity Equation and Reynolds's Equation for modeling a capacitive micro-switch. They employed a non-linear Newmark time integration scheme for the mechanical Equations and a trapezoidal rule for the fluid Equations. The above mentioned models attempt to solve the coupled problem, though not in a single step. The geometry modeled is also limited to 1D beam type structures.

These methods, though accurate, are cumbersome and involve iterative or staggered approaches. We propose a single step methodology to solve the coupled fluid- structure squeeze film problem. We use the 3D elasticcity Equation, thus not restricting ourselves to any particular geometry, and couple it with the 2D Reynolds Equation for squeeze film. A single step "monolith" approach [10] is presented to solve the coupled problem. The numerical results show good agreement with published experimental data and existing analytical solutions.

\section{Numerical Modeling}

The problem at hand involves solving for pressure on the vibrating plate due to the squeezed film, taking into account the elasticity of the plate. Thus the problem involves solution of the Reynolds Equation for the fluid domain coupled with the 3D elasticity Equation for the structural domain. In our finite element model, the air gap is treated as a $2 \mathrm{D}$ layer and the structural domain is modeled in three dimensions. The element used for modeling the structural domain is $3 \mathrm{D}, 27$ node brick element. The "wet" face of the 3D element is treated as the fluid domain. Thus the relevant integrals for the fluid domain are evaluated over the corresponding 9 noded "wet" surface of the 3D, 27 node brick element.

\subsection{Variational Formulation for the Fluid Domain}

The linearized Reynolds Equation is given as follows [2],

$$
\frac{h_{0}^{3}}{12 \mu_{\mathrm{eff}}}\left(\frac{\partial^{2} P}{\partial x^{2}}+\frac{\partial^{2} P}{\partial y^{2}}\right)=\frac{h_{0}}{P_{\mathrm{a}}} \frac{\partial P}{\partial t}+\frac{\partial H}{\partial t},
$$

where $\mu_{\text {eff }}$ is the effective viscosity, $h_{0}$ is the initial air gap, $P_{a}$ is the ambient air pressure, $P$ is the fluid pressure (perturbed about $P_{\mathrm{a}}$ ) and $H$ is the air gap (perturbed about $h_{0}$ ). The last term on the right hand side of the above Equation couples the structure and the fluid domain. Substituting for harmonic solution $P=\tilde{p} e^{j \omega t}$ and $H=\tilde{u}_{z} e^{j \omega t}$ in Equation (1) and considering $p_{\delta}$ as variation of $\tilde{p}$ in a weighted integral sense we have,

$$
\int_{\Omega}\left(\frac{h_{0}^{3}}{12 \mu_{\text {eff }}} \nabla^{2} \tilde{p}-\frac{h_{0} j \omega}{P_{\text {a }}} \tilde{p}-j \omega \tilde{u}_{z}\right) p_{\delta} d \Omega=0 .
$$

For the fluid domain we have $\tilde{p}=0$ on the open borders and $\frac{\partial \tilde{p}}{\partial \hat{n}}=0$ on the closed borders. After doing integration by parts and implementing the above boundary conditions, we get the governing Equation for fluid domain as,

$$
\begin{aligned}
& \int_{\Omega}\left(\frac{h_{0}^{3}}{12 \mu_{\mathrm{eff}}} \nabla \tilde{p} \cdot \nabla p_{\delta}\right) d \Omega+\int_{\Omega}\left(\frac{h_{0} j \omega}{P_{\mathrm{a}}} \tilde{p} p_{\delta}\right) d \Omega \\
& +\int_{\Omega} j \omega \tilde{u}_{z} p_{\delta} d \Omega=0
\end{aligned}
$$




\subsection{FEM Formulation for the Fluid Domain}

For the FEM formulation we use 9 noded quadrilateral elements for 2D fluid domain. Pressure, its variation and $\tilde{u}_{z}$ at any point are obtained from interpolation of the corresponding nodal values using the following relationships.

$$
\tilde{p}=N_{p} \hat{\boldsymbol{p}}=\left[\begin{array}{llll}
N_{1} & N_{2} & \cdots & N_{9}
\end{array}\right]\left[\begin{array}{c}
p_{1} \\
p_{2} \\
\vdots \\
p_{9}
\end{array}\right]
$$$$
p_{\delta}=\boldsymbol{N}_{p} \hat{\boldsymbol{p}}_{\delta}
$$

$$
\tilde{u}_{z}=N_{u_{z}} \hat{\boldsymbol{u}}=\left[\begin{array}{lllllll}
0 & 0 & N_{1} & \cdots & 0 & 0 & N_{9}
\end{array}\right]\left[\begin{array}{c}
u_{1} \\
v_{1} \\
w_{1} \\
\vdots \\
u_{9} \\
v_{9} \\
w_{9}
\end{array}\right] .
$$

Pressure gradient can be expressed as

$$
\nabla \tilde{p}=\boldsymbol{B}_{p} \hat{\boldsymbol{p}},
$$

where

$$
\boldsymbol{B}_{p}=\left[\begin{array}{cccc}
\frac{\partial N_{1}}{\partial x} & \frac{\partial N_{2}}{\partial x} & \cdots & \frac{\partial N_{9}}{\partial x} \\
\frac{\partial N_{1}}{\partial y} & \frac{\partial N_{2}}{\partial y} & \cdots & \frac{\partial N_{9}}{\partial y}
\end{array}\right]
$$

Similarly we have,

$$
\nabla p_{\delta}=\boldsymbol{B}_{p} \hat{\boldsymbol{p}}_{\delta}
$$

where $\boldsymbol{N}_{p}$ is $(1 \times 9), \hat{\boldsymbol{p}}$ is $(9 \times 1), \quad \hat{\boldsymbol{p}}_{\delta}$ is $(9 \times 1) \quad \boldsymbol{B}_{p}$ is $(2 \times 9), \quad \boldsymbol{N}_{u_{z}}$ is $(1 \times 27)$ and $\hat{\boldsymbol{u}}$ is $(27 \times 1)$.

Substituting Equations (4), (5), (6), (7) and (9) in Equation (3) and using arbitrariness of $\hat{\boldsymbol{p}}_{\delta}$ we get,

$$
\begin{aligned}
& {\left[\frac{h_{0}^{3}}{12 \mu_{\mathrm{eff}}} \int_{\Omega} \boldsymbol{B}_{\boldsymbol{p}}^{T} \boldsymbol{B}_{\boldsymbol{p}} d \Omega+\frac{j \omega h_{0}}{P_{\mathrm{a}}} \int_{\Omega} \boldsymbol{N}_{\boldsymbol{p}}^{T} \boldsymbol{N}_{\boldsymbol{p}} d \Omega\right] \hat{\boldsymbol{p}}} \\
& +\left[j \omega \int_{\Omega} \boldsymbol{N}_{\boldsymbol{p}}^{T} \boldsymbol{N}_{\boldsymbol{u}_{\boldsymbol{z}}} d \Omega\right] \hat{\boldsymbol{u}}=0 .
\end{aligned}
$$

\subsection{FEM Formulation for the Structure}

We have the variational statement for dynamic structural problem without anybody force as:

$$
\int_{\Omega} \tau(\boldsymbol{u}): \boldsymbol{\varepsilon}\left(\boldsymbol{u}_{\delta}\right) d \Omega+\int_{\Omega} \rho \ddot{\boldsymbol{u}} \boldsymbol{u}_{\delta} d \Omega=\int_{\Gamma_{t}} \boldsymbol{u}_{\delta} \cdot \overline{\boldsymbol{t}} d \Gamma,
$$

where $\rho$ is density, $\boldsymbol{u}$ is displacement, $\boldsymbol{u}_{\delta}$ is its variation, $\boldsymbol{\tau}(\boldsymbol{u})$ is stress, $\overline{\boldsymbol{t}}$ is prescribed traction over the boundary $\Gamma_{\mathrm{t}}$ and $\varepsilon\left(\boldsymbol{u}_{\delta}\right)$ is given by

$$
\boldsymbol{\varepsilon}\left(\boldsymbol{u}_{\delta}\right)=\frac{1}{2}\left[\left(\nabla \boldsymbol{u}_{\delta}\right)+\left(\nabla \boldsymbol{u}_{\delta}\right)^{T}\right] .
$$

Here,

$$
\tau(\boldsymbol{u}): \varepsilon\left(\boldsymbol{u}_{\delta}\right)=\tau_{\mathrm{ij}}(\boldsymbol{u}): \varepsilon_{\mathrm{ij}}\left(\boldsymbol{u}_{\delta}\right),
$$

with the summation convention over repeated indices.

For coupled squeeze film damping problem with structural interaction, the wet surface (the surface which constitutes the 2D fluid domain) is subjected to prescribed traction $\overline{\boldsymbol{t}}=-\tilde{\boldsymbol{p}} \hat{\boldsymbol{n}}$, then Equation (11) can be written as

$$
\begin{aligned}
& \int_{\Omega} \boldsymbol{\tau}(\boldsymbol{u}): \boldsymbol{\varepsilon}\left(\boldsymbol{u}_{\delta}\right) d \Omega+\int_{\Omega} \rho \ddot{\boldsymbol{u}} \boldsymbol{u}_{\delta} d \Omega+\int_{\Gamma_{\mathrm{wet}}} \tilde{p} \boldsymbol{u}_{\delta} \cdot \hat{\boldsymbol{n}} d \Gamma \\
& =\int_{\Gamma_{\mathrm{t}}} \boldsymbol{u}_{\delta} \cdot \overline{\boldsymbol{t}} d \Gamma,
\end{aligned}
$$

where the last term on the left hand side signifies coupling effect of the fluid over the structural domain. The standard FEM discretization for displacement and other quantities are

$$
\begin{aligned}
& \boldsymbol{u}=N_{u} \hat{\boldsymbol{u}}, \\
& \boldsymbol{u}_{\delta}=N_{u} \hat{\boldsymbol{u}}_{\delta}, \\
& \tau(\boldsymbol{u})=C B_{u} \hat{\boldsymbol{u}}, \\
& \boldsymbol{\varepsilon}(\boldsymbol{u})=\boldsymbol{B}_{u} \hat{\boldsymbol{u}}, \\
& \boldsymbol{\varepsilon}\left(\boldsymbol{u}_{\delta}\right)=\boldsymbol{B}_{u} \hat{\boldsymbol{u}}_{\delta} .
\end{aligned}
$$

Substituting above relations in Equation (12) and using arbitrariness of $\boldsymbol{u}_{\delta}$ we have the discretized Equation for the structural domain as

$$
\begin{aligned}
& {\left[\int_{\Omega} \boldsymbol{B}_{u}^{T} \boldsymbol{C} \boldsymbol{B}_{u} d \Omega\right] \hat{\boldsymbol{u}}+\left[\int_{\Omega} \rho \boldsymbol{N}_{u}^{T} \boldsymbol{N}_{u} d \Omega\right] \ddot{\hat{\boldsymbol{u}}}} \\
& +\left[\int_{\Gamma_{\mathrm{wet}}} \rho \boldsymbol{N}_{u}^{T} \hat{\boldsymbol{n}} \boldsymbol{N}_{\boldsymbol{p}} d \Gamma\right] \hat{\boldsymbol{p}}=\int_{\Gamma_{\mathrm{t}}} \boldsymbol{N}_{u}^{T} \overline{\boldsymbol{t}} d \Gamma .
\end{aligned}
$$

\subsection{Coupled FEM Formulation}

For the coupled problem at hand the 2D fluid domain corresponds to the "wet" surface of the structural domain. Thus coupling the fluid and structure domains we have (combining Equations (10) and (13))

$$
\left[\begin{array}{ll}
\boldsymbol{K}_{\mathrm{uu}} & \boldsymbol{K}_{\mathrm{up}} \\
\boldsymbol{K}_{\mathrm{pu}} & \boldsymbol{K}_{\mathrm{pp}}
\end{array}\right]\left\{\begin{array}{c}
\hat{\boldsymbol{u}} \\
\hat{\boldsymbol{p}}
\end{array}\right\}=\left\{\begin{array}{c}
\boldsymbol{f}_{\mathrm{u}} \\
\boldsymbol{0}
\end{array}\right\},
$$




$$
\begin{aligned}
& \boldsymbol{K}_{u \boldsymbol{u}}=-\omega^{2} \boldsymbol{M}_{\boldsymbol{u}}+\boldsymbol{K}_{u}, \\
& \boldsymbol{M}_{u}=\int_{\Omega} \rho \boldsymbol{N}_{u}^{T} \boldsymbol{N}_{u} d \Omega, \\
& \boldsymbol{K}_{u}=\int_{\Omega} \boldsymbol{B}_{u}^{T} \boldsymbol{C} \boldsymbol{B}_{u} d \Omega, \\
& \boldsymbol{K}_{u p}=\int_{\Gamma_{\mathrm{wet}}} \boldsymbol{N}_{u}^{T} \hat{\boldsymbol{n}} \boldsymbol{N}_{p} d \Gamma, \\
& \boldsymbol{f}_{u}=\int_{\Gamma_{\mathrm{t}}} \boldsymbol{N}_{u}^{T} \overline{\boldsymbol{t}} d \Gamma, \\
& \boldsymbol{K}_{p p}=\frac{h_{0}^{3}}{12 \mu_{\mathrm{eff}}} \int_{\Gamma_{\mathrm{wet}}} \boldsymbol{B}_{p}^{T} \boldsymbol{B}_{p} d \Gamma+\frac{j \omega h_{0}}{P_{\mathrm{a}}} \int_{\Gamma_{\mathrm{wet}}} \boldsymbol{N}_{p}^{T} \boldsymbol{N}_{p} d \Gamma, \\
& \boldsymbol{K}_{p u}=j \omega \int_{\Gamma_{\mathrm{wet}}} \boldsymbol{N}_{p}^{T} \boldsymbol{N}_{p} d \Gamma .
\end{aligned}
$$

We have used 27 noded brick element for the structural domain, whose wet surface (consisting of 9 noded square face) is modelled as the 2D fluid domain.

\section{Results and Discussion}

For validation of our FEM results we have compared our numerical results with analytical solutions reported by Siddartha et al. [11,12], as well as experimental results from work by Pandey et al. [8].

\subsection{Modeling a Varying Flow Boundary Elastic Microplate}

Siddartha et al. $[11,12]$ studied the effect of varying flow boundary conditions on the squeeze film stiffness and damping for an all sides clamped micro-plate. The plate is considered to vibrate in its fundamental mode which imparts the flexibility effect. Analytical expressions have been derived for stiffness and damping forces on the plate (clamped at all sides) due to the trapped air film (subjected to different flow boundaries). We use $4 \times 4$ mesh for FEM modeling of the plate. We design two representative flow boundary conditions with our numerical scheme, namely the all four sides open ("OOOO") and the two opposite sides closed ("OCOC") cases. The plate is subjected to harmonic displacement boundary condition corresponding to its first mode shape. The resulting pressure distribution is integrated over the wet surface to get the force on the moving plate. The squeeze film spring $\left(F_{s}\right)$ and damping $\left(F_{d}\right)$ forces are obtained from the real and imaginary component of the resultant force respectively. The forces are non dimensionalised (see [11]) and plotted against a non dimensional parameter $\sigma$, directly related to the frequency of harmonic excitation as follows, $\sigma=12 \mu_{\text {eff }} \omega L^{2} / p_{0} h_{0}^{2}$, where $\mu_{\text {eff }}$ is the effective viscosity [13], $\omega$ is the harmonic excitation frequency, $L$ is the plate side dimension, $p_{0}$ is the ambient pressure and $h_{0}$ is the initial air gap. Figure 2 shows the plots for $F_{s}$ and $F_{d}$ for the "OOOO" case and Figure 3 shows the same for the "OCOC" case. We see from these plots that the numerical stiffness and damping forces are in close agreement with the analytical results for both the flow boundary cases studied. We also note that the two methods show good agreement at both high and low $\sigma$ values (thus high and low frequencies). The deviation between the numerical and analytical results have been found to be less than $2 \%$ for both the flow boundary conditions studied here.

\subsection{Modeling a Cantilever}

In order to compare our results with experimental data we have modeled a cantilever beam as per dimensions

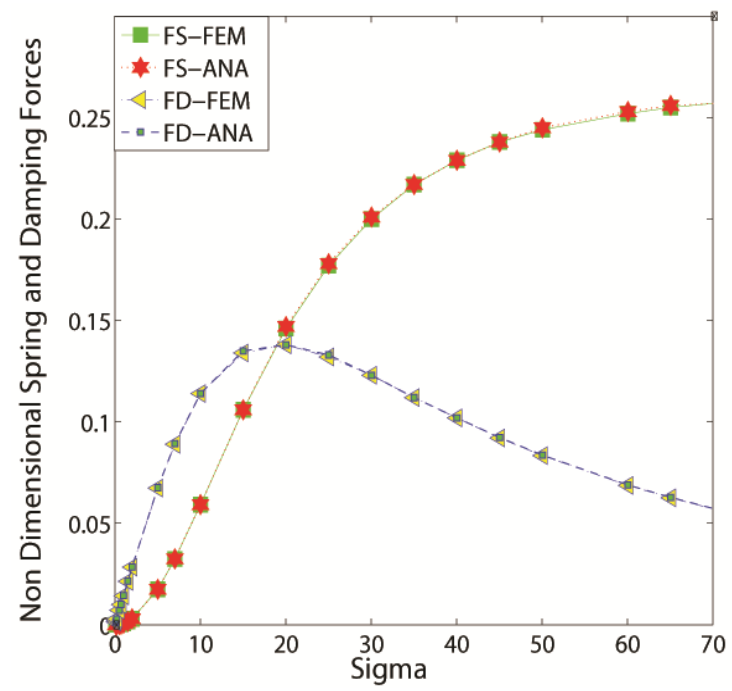

Figure 2. Spring and damping forces vs sigma for "OOOO" configuration.

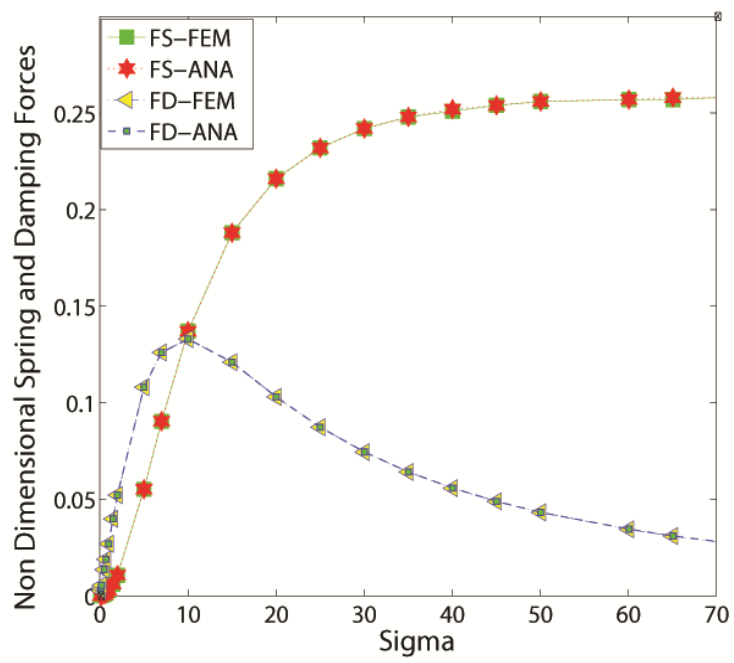

Figure 3. Spring and damping forces vs sigma for "OCOC" configuration. 
mentioned in the work of Pandey et al. [8]. We compare the numerically obtained Quality factors $(Q)$ for the first three modes of vibration as well as the effect of aspect ratio on the quality factor of the beam for the first mode of vibration. The beam modeled is of length $350 \mu \mathrm{m}$, width $22 \mu \mathrm{m}$, thickness $4 \mu \mathrm{m}$, with an initial air gap of $1.4 \mu \mathrm{m}$. The beam material is polysilicon with density $2330 \mathrm{Kg} / \mathrm{m}^{3}$, Young's Modulus $160 \mathrm{GPa}$ and Poisson's ratio 0.22 . Air is considered to be the fluid medium with the relevant property values (under standard temperature and pressure ) as follows: density $\rho_{\text {air }}=1.2 \mathrm{Kg} / \mathrm{m}^{3}$, dynamic viscosity $\mu_{\text {air }}=1.8 \times 10^{-5} \mathrm{~N} . \mathrm{s} / \mathrm{m}^{2}$, and ambient pressure $p_{\mathrm{a}}=1.013 \times 10^{5} \mathrm{~Pa}$. In our simulations we have subjected the cantilever to a sinusoidal voltage of $1.5 \mathrm{~V}$, which is well below the pull-in voltage of $6 \mathrm{~V}$ for the given cantilever. The input voltage has been applied as an electrostatic pressure load of magnitude $5.08 \mathrm{~N} / \mathrm{m}^{2}$ to our FEM model for the cantilever beam, considering a parallel plate capacitor with small displacement approximation [14]. The beam tip velocities have been obtained from the simulations and normalized with respect to the applied voltage and plotted against frequency. The frequency response so obtained is shown in Figure 4. The plot shows three distinct peaks corresponding to the first three modes, and is in close agreement with a similar plot reported by Pandey et al. [8]. $Q$ factors for different modes are obtained using half power method from the frequency response plot. Convergence study of the $Q$ factor (Table 1) has been done using three levels of mesh refinement considering a very fine mesh $(100 \times 6 \times 4)$ result as our benchmark. $Q$ factors for sufficiently fine mesh $(40 \times 5 \times 4)$ are compared with published results from Pandey et al. [8] in Table 2. We see that the data from the numerical simulations are in good agreement with published experimental and numerical results. We

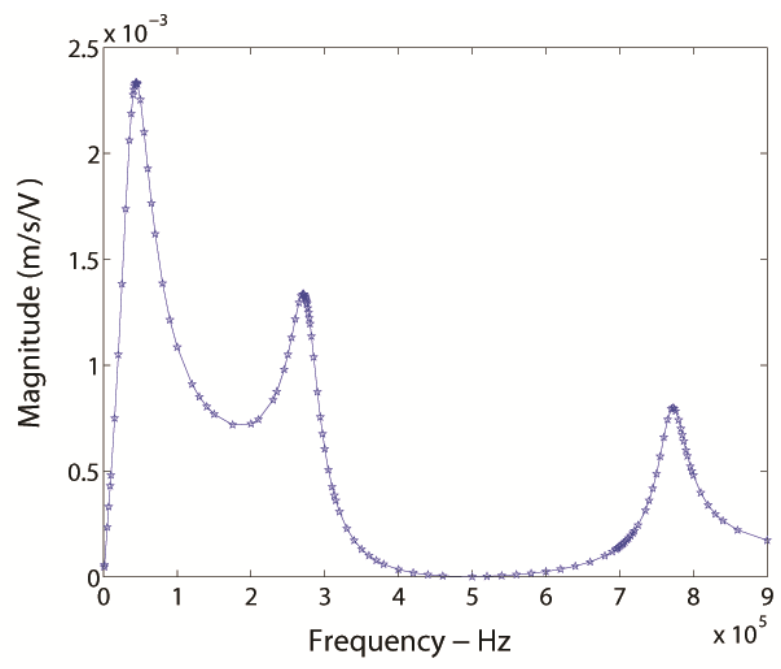

Figure 4. Frequency response of a cantilever beam of length $350 \mu \mathrm{m}$, width $22 \mu \mathrm{m}$ and thickness $4 \mu \mathrm{m}$. further studied beams with varying aspect ratios. We considered beams with lengths varying from $150 \mu \mathrm{m}$ to $350 \mu \mathrm{m}$, having a constant width of $22 \mu \mathrm{m}$ and thickness $4 \mu \mathrm{m}$. Only the first mode of vibration is considered in this study. Comparative values of $Q$ factors for the different beams $(40 \times 5 \times 4$ mesh $)$ are presented in Table 3 . Our simulation results show a deviation of less than $10 \%$ from the reported experimental data.

\section{Conclusion}

We have discussed an FEM formulation to solve the coupled fluid-structure squeeze film problem without resorting to iterative solutions. Our results show good agreement with experimental data available from the literature. Our numerical scheme is seen to give good results for varying aspect ratio structures with dimensions below $100 \mu \mathrm{m}$. The proposed scheme can be further used as a design tool for modeling and simulation of the dynamic response of vibratory MEMS devices such as capacitive microphones, RF (Radio Frequency) MEMS switches, etc., for which accurate knowledge of the $Q$

Table 1. Convergence study for the first two modes of a cantilever beam of length $350 \mu \mathrm{m}$, width $22 \mu \mathrm{m}$.

\begin{tabular}{ccccc}
\hline \multirow{2}{*}{ Modes } & \multicolumn{4}{c}{ Q factors for FEM Mesh (length $\times$ width $\times$ thickness) } \\
\cline { 2 - 5 } & $100 \times 6 \times 4$ & $40 \times 5 \times 4$ & $30 \times 3 \times 2$ & $20 \times 3 \times 2$ \\
\hline 1 & 1.097 & 1.095 & 1.093 & 1.10 \\
2 & 5.842 & 5.849 & 5.891 & 5.908 \\
\hline
\end{tabular}

Table 2. $Q$ factor comparison for the first three modes of a cantilever beam of length $350 \mu \mathrm{m}$, width $22 \mu \mathrm{m}$.

\begin{tabular}{cccc}
\hline \multirow{2}{*}{ Modes } & \multicolumn{3}{c}{$Q$ factors comparison } \\
\cline { 2 - 4 } & $Q_{\text {EXP }}$ & $Q_{\text {ANSY }}$ & $Q_{\text {FEM }}$ \\
\hline 1 & 1.20 & 1.11 & 1.095 \\
2 & 7.58 & 6.94 & 5.849 \\
3 & 18.52 & 20.0 & 20.379 \\
\hline
\end{tabular}

Table 3. $Q$ factor comparison for beam of width $22 \mu \mathrm{m}$ with varying lengths.

\begin{tabular}{cccc}
\hline \multirow{2}{*}{ Length $(\mu \mathrm{m})$} & \multicolumn{2}{c}{$Q$ factors } & \% Deviation \\
\cline { 2 - 3 } & $Q_{\mathrm{EXP}}$ & $Q_{\mathrm{FEM}}$ & \\
\hline 150 & 7.04 & 6.83 & 2.98 \\
200 & 4.0 & 3.66 & 8.5 \\
250 & 2.49 & 2.26 & 9.24 \\
300 & 1.56 & 1.53 & 2.05 \\
350 & 1.2 & 1.09 & 9.17 \\
\hline
\end{tabular}


factor is critical for design. With this methodology one can directly couple the elasticity effect of the structure with the fluid flow and need not limit oneself to 1D geometries.

\section{Acknowledgements}

This work is partially supported by NPMASS grant for computational micro-systems. The authors acknowledge the support from CoNE Lab of the Center for Nano Science and Engineering at IISC.

\section{REFERENCES}

[1] M. Bao and H Yang, "Squeeze Film Air Damping in MEMS," Sensors and Actuators A, Vol. 136, No. 1, 2007 , pp. 3-27. http://dx.doi.org/10.1016/j.sna.2007.01.008

[2] R. Pratap, S. Mohite and A. K. Pandey, "Squeeze Film Effects in MEMS Devices," Journal of the Indian Institute of Science, Vol. 87, No. 1, 2007, pp. 75-94. http://eprints.iisc.ernet.in/id/eprint/12328

[3] J. J. Blech, "On Isothermal Squeeze Films," Journal of Lubrication Technology, Vol. 105 No. 4, 1983, pp. 615620. http://dx.doi.org/10.1115/1.3254692

[4] R. B. Darling, C. Hivick and J. Xu, "Compact Analytical Models for Squeeze Film Damping with Abitrary Venting Conditions," International Conference on Solid State Sensors and Actuators, Vol. 2, 1997, pp. 1113-1116. http://dx.doi.org/10.1109/SENSOR.1997.635397

[5] E. S. Hung and S. D. Senturia, "Generating Efficient Dynamical Models for Microelectromechanical Systems from a Few Finite-Element Simulation Runs," Journal of Microelectromechanical Systems, Vol. 8, No. 3, 1999, pp. 280-289. http://dx.doi.org/10.1109/84.788632

[6] B. McCarthy, G. G. Adams, N. E. McGruer and D. Potter, "A Dynamic Model, Including Contact Bounce, of an Electrostatically Actuated Microswitch," Journal of Microelectromechanical Systems, Vol. 11, No. 3, 2002, pp. 276-283.
http://dx.doi.org/10.1109/JMEMS.2002.1007406

[7] M. I. Younis and A. H. Nayfeh, "Simulation of SqueezeFilm Damping of Microplates Actuated by Large Electrostatic Load," Journal of Computational and Nonlinear Dynamics, Vol. 2, No. 3, 2007, pp. 232-240. http://dx.doi.org/10.1115/1.2727491

[8] A. K. Pandey and R. Pratap, "Effect of Flexural Modes on Squeeze Film Damping in MEMS Cantilever Resonators," Journal of Micromechanics and Microengineering, Vol. 17 No. 12 , 2007, pp. 2475-2484. ttp://dx.doi.org/10.1088/0960-1317/17/12/013

[9] S. D. A. Hannot and D. J. Rixen, "Coupling Plate Deformation, Electrostatic Actuation and Squeeze Film Damping in a FEM Model of a Micro Switch," International Conference on Computational Methods for Coupled Problems in Science and Engineering, Barcelona, 2009.

[10] C. S. Jog, "An Outward-Wave-Favouring Finite ElementBased Strategy for Exterior Acoustical Problems," International Journal of Acoustics and Vibration, Vol. 18, No. 1, 2013, pp. 27-38

http://eprints.iisc.ernet.in/id/eprint/46436

[11] S. Patra, A. Roychowdhury and R. Pratap, "Effect of Fluid Flow Boundary Conditions on Squeeze Film Parameters of Planar MEMS Structures," Applied Mathematics Modelling, AMM 14573, Unpublished.

[12] S. Patra, "Effect of Rarefaction, Flexibility and Restrictive Flow Boundary Conditions on Squeeze Film Flow of MEMS Devices," Master's Thesis, Indian Institute of Science, Bangalore, 2011.

[13] T. Veijola, H. Kuisma, J. Lahdenperä and T. Ryhänen, "Equivalent-Circuit Model of the Squeezed Gas Film in a Silicon Accelerometer," Sensors and Actuators A: Physical, Vol. 48, No. 43, 1995, pp. 239-248, http://dx.doi.org/10.1016/0924-4247(95)00995-7

[14] E. Saucedo-Flores, R. Ruelas, M. Florer and I. C. Chiao, "Study of the Pull in Voltage for MEMS Parallel Plate Capacitor Actuators," Materials Research Society Fall Meeting, Boston, 1-5 December 2003. 